Article

\title{
Transcriptome-Wide Single Nucleotide Polymorphisms (SNPs) for Abalone (Haliotis midae): Validation and Application Using GoldenGate Medium-Throughput Genotyping Assays
}

\author{
Aletta Bester-Van Der Merwe *, Sonja Blaauw, Jana Du Plessis and Rouvay Roodt-Wilding \\ Molecular Breeding and Biodiversity Group, Department of Genetics, Faculty of Agrisciences, \\ Stellenbosch University, Private Bag X1, Matieland 7602, South Africa; \\ E-Mails: me.sonja.blaauw@gmail.com (S.B.); jdup27@gmail.com (J.P.); roodt@sun.ac.za (R.R.-W.) \\ * Author to whom correspondence should be addressed; E-Mail: aeb@sun.ac.za; \\ Tel.: +27-21-808-5835; Fax: +27-21-808-5833.
}

Received: 26 July 2013; in revised form: 26 August 2013 / Accepted: 5 September 2013 /

Published: 23 September 2013

\begin{abstract}
Haliotis midae is one of the most valuable commercial abalone species in the world, but is highly vulnerable, due to exploitation, habitat destruction and predation. In order to preserve wild and cultured stocks, genetic management and improvement of the species has become crucial. Fundamental to this is the availability and employment of molecular markers, such as microsatellites and single nucleotide (SNPs). Transcriptome sequences generated through sequencing-by-synthesis technology were utilized for the in vitro and in silico identification of 505 putative SNPs from a total of 316 selected contigs. A subset of 234 SNPs were further validated and characterized in wild and cultured abalone using two Illumina GoldenGate genotyping assays. Combined with VeraCode technology, this genotyping platform yielded a $65 \%-69 \%$ conversion rate (percentage polymorphic markers) with a global genotyping success rate of $76 \%-85 \%$ and provided a viable means for validating SNP markers in a non-model species. The utility of 31 of the validated SNPs in population structure analysis was confirmed, while a large number of SNPs (174) were shown to be informative and are, thus, good candidates for linkage map construction. The non-synonymous SNPs (50) located in coding regions of genes that showed similarities with known proteins will also be useful for genetic applications, such as the marker-assisted selection of genes of relevance to abalone aquaculture.
\end{abstract}


Keywords: abalone; Haliotis midae; SNP validation; transcriptome; GoldenGate assay; medium-throughput genotyping

\section{Introduction}

The South African abalone, Haliotis midae, is a highly valuable marine resource and one of almost 30 commercially viable abalone species found worldwide [1]. The South African abalone industry, the largest outside Asia, currently has a total output of 1015.44 metric tons, valued at 355 million South African Rand (ZAR) [2]. In order to supply the growing international market and to ensure the sustainability of the industry, effective farm management practices, including the employment of molecular markers in genetic management programs, are vital [3]. To date, mainly microsatellite markers (274) have been developed for H. midae using various enrichment and in silico techniques [4-7]. Although these markers have already proven to be very useful, they have limitations, including development time, size homoplasy and the presence of null alleles [4]. The focus has therefore shifted towards the development of single nucleotide polymorphisms (SNPs) with 40 SNPs isolated thus far [8,9]. These markers have become increasingly popular in recent years, due to their abundance in genomes, the ease of genotyping and the reduction in development costs [10]. The increase of SNP development in aquaculture species is evident in reports on Atlantic cod (Gadus morhua) [11,12], Japanese scallop (Patinopecten yessoensis) [13], Atlantic salmon (Salmo salar) [14,15], Channel catfish (Ictalurus punctatus) [16] and, also, species of abalone, such as Pacific abalone (Haliotis discus hannai) [17,18].

In the case of $H$. midae, as in many other non-model organisms, no complete genome map is currently available. Transcriptomic data (in the form of expressed sequence tags (ESTs)), however, serve as a viable source for SNP discovery [8,19-21], facilitating an easier and more cost-effective means of generating genomic resources in non-model organisms [22]. Despite limitations, such as inferring intron positions and genomic gene order, the use of transcribed sequences for marker development proves advantageous, since such markers are directly associated with genes and could be very useful for gene-associated mapping, the identification of causative genes and interspecific transferability between closely related species; an important consideration, especially in non-model species, where knowledge of the genome is limited [23].

Single nucleotide polymorphism isolation procedures have improved greatly, due to advances in DNA sequence technology, and, at present, most commonly include the utilization of whole genome or transcriptome next generation sequencing (NGS) data. The NGS platforms expedite sequence data generation by increasing the production of sequence data to several thousand megabase pairs $(\mathrm{Mb})[24,25]$, and the generated reads allow for efficient assembly of contigs [26,27]. Next generation sequencing has paved the way for sequencing, genotyping and high-throughput marker discovery at an affordable rate [28]. Mining of SNPs from NGS-generated ESTs mainly involves creating, clustering and assembling the generated ESTs, followed by SNP identification by means of in vitro or in silico approaches [29]. In vitro detection of SNPs involves re-sequencing of targeted ESTs to identify nucleotide variations, whereas in silico detection refers to the use of bioinformatic pipelines to 
identify polymorphisms [30,31]. Large-scale data generation methods, such as NGS, coupled with high-throughput genotyping techniques allow for far more efficient means of SNP development and characterization than was previously possible.

Currently, various high-throughput genotyping platforms exist, but only a few are suitable for medium-throughput genotyping, which is preferred for non-model species [32]. In this study, medium-throughput genotyping for SNP characterization was performed using the Illumina GoldenGate genotyping assay with VeraCode technology on the BeadXpress platform. The GoldenGate assay includes locus identification by means of hybridization, enzymatic allele discrimination and exponential amplification of target sequences with the use of three assay oligonucleotides for each SNP [33]. The first two oligonucleotides are allele-specific oligonucleotides (ASO), while the third oligonucleotide, the locus-specific oligonucleotide (LSO), is complementary to the sequence of interest, thus allowing for hybridization downstream from the SNP. Various degrees of multiplexing can be applied to minimize cost and time [34]. The VeraCode technology employs silica glass microbeads inscribed with digital holographic barcodes, which act as solid substrates in solution [35]. All three oligonucleotides are complementary to the universal primers, but the LSO also has a unique address sequence that is complementary to a specific VeraCode bead. The final component is the BeadXpress Reader, a platform with a dual-color laser that scans the microbeads to identify the unique code within each bead.

In this study, we focused on the development of SNPs from transcriptome data previously described for $H$. midae following two bioinformatic pipelines. The EST data formed the basis for in vitro and in silico SNP discovery. Primer efficiency and genotyping success was evaluated by characterization of two GoldenGate assays in individuals from wild and cultured H. midae populations. Downstream applications of successfully genotyped SNPs were also assessed for both GoldenGate assays, and these results form part of an integrative research effort with regards to genetic characterization and improvement of South African abalone. The 48-plex GoldenGate assay (Plex-48) was employed to assess population differentiation between six H. midae populations, and a second 192-plex GoldenGate assay (Plex-192) was used to illustrate and test the utility of SNP markers in conjunction with microsatellite markers for linkage map construction in eight $H$. midae full-sib families.

\section{Results and Discussion}

\subsection{Transcriptome Data and SNP Discovery}

The Velvet assembly of transcriptome data utilized in the first bioinformatics analysis yielded 30,689 contigs with a minimum length of $80 \mathrm{bp}$ and an average length of $276 \mathrm{bp}$. The total number of contigs that resulted from the CLC Genomics Workbench de novo assembly was 22,761, with an average length of $260 \mathrm{bp}$ and an average contig coverage of 400 reads/contig. For the in vitro SNP detection via re-sequencing of 58 selected contigs, a total of $66 \%$ of the optimized PCR amplicons yielded trace quality adequate for putative SNP discovery. The in vitro primer success rate (primers that amplified successfully) observed correlated well with similar studies on aquaculture species, including that of the Eastern oyster (Crassostrea virginica) (69\%) [36], Pearl mussel (Hyriopsis cumingii) (58\%) [37] and Pacific abalone (Haliotis discus hannai) (67.3\%) [17]. The general consensus is that 
stringent parameters and the knowledge of intron-exon boundaries are important considerations for designing primers when utilizing the in vitro approach [17,38]. A study in Pacific oyster (Crassostrea gigas) also showed a marked ( $30 \%)$ increase in primer success rate, by designing primers where the reverse primer is situated in the 3' untranslated region (UTR) [39]. A similar rationale was followed in the current study on the premise that introns are highly infrequent in the 3'-UTR [40]. For the in silico detection approach using the SNP detection module in CLC Genomics Workbench, 958 assembled contigs containing 3645 putative SNPs were identified. Following the in vitro and in silico detection approaches, a subset of 505 putative SNPs (105 in vitro, 400 in silico) was selected. This amounted to approximately one SNP every $550 \mathrm{bp}$, which is a much lower SNP frequency than previously obtained in $H$. midae (one SNP every $\sim 150 \mathrm{bp}$; [8,9]) and in other Haliotid species (1 SNP every $\sim 50 \mathrm{bp} ;[17,38]$ ). This could possibly be ascribed to the stringent parameters that were set for putative SNP calling, such as the absence of polymorphisms in the $60 \mathrm{bp}$ flanking sequences required for the GoldenGate assays. Studies in catfish found an increase in SNP frequency coupled with an increase of contig size [41], but no association between the fragment length and the number of putative SNPs was observed for this study. A total of $65(62 \%)$ transitions and $38(36 \%)$ transversions were observed for the in vitro SNPs, giving an observed transition to transversion (ts:tv) ratio of 1.71 , while $253(63 \%)$ transitions and $145(36 \%)$ transversions for the in silico SNPs, representing a ts:tv ratio of 1.74 (Table 1). Although the overall ts:tv ratio of 1.74 observed in the current study was slightly higher than previously obtained for $H$. midae [7,11,12], a high ts:tv ratio in general is a good measure for a low frequency of false positives in SNP development and confirmed a high validation rate for the selected SNPs in this study [42].

Table 1. Summary of the variants of the putative single nucleotide polymorphisms (SNPs) detected in vitro and in silico for $H$. midae following two bioinformatic pipelines.

\begin{tabular}{lcc}
\hline & In vitro (Velvet assembly) & In silico (CLC assembly) \\
\hline Number of contigs & 58 & 256 \\
Number of putative SNPs & 105 & 400 \\
Transversions: & & \\
$\mathrm{A} / \mathrm{T}$ & $15(14.3 \%)$ & $52(13.0 \%)$ \\
$\mathrm{A} / \mathrm{C}$ & $7(6.7 \%)$ & $35(8.8 \%)$ \\
$\mathrm{C} / \mathrm{G}$ & $10(9.5 \%)$ & $15(3.8 \%)$ \\
$\mathrm{T} / \mathrm{G}$ & $6(5.7 \%)$ & $43(10.8 \%)$ \\
Transitions: & & \\
$\mathrm{A} / \mathrm{G}$ & $35(33.3 \%)$ & $124(31.0 \%)$ \\
$\mathrm{T} / \mathrm{C}$ & $30(28.6 \%)$ & $129(32.3 \%)$ \\
Other & $2(1.9 \%)$ & $2(0.5 \%)$ \\
\hline
\end{tabular}

\subsection{SNP Performance}

Due to the various SNP isolation methods used in this study, it was necessary to evaluate the different approaches in terms of marker performance. The SNPs evaluated in Plex-48 were isolated in four different ways: (1) directly from a cDNA library via a targeted EST approach (8\%), (2) from microsatellite flanking regions (17\%), (3) from EST contigs and re-sequencing (in vitro: 50\%) and 
(4) via the CLC workbench approach (in silico: 25\%). The loci included in Plex-192 were all detected using the CLC workbench SNP detection utility. It was found that the majority (57\%) of SNPs that failed to genotype were identified from microsatellite flanking regions. This failure could be due to the hyper-variability of microsatellite flanking regions and, consequently, the possibility that primers are binding in variable regions [9]. The success of SNPs isolated in silico depends mostly on the species, the representative sample used for generating data, as well as the quality of the sequencing data $[10,41,43]$. In this study, sequencing data with high representation and depth [44] was used in order to identify candidate SNPs with high confidence, and even though $17 \%$ of the in silico markers from Plex-48 and $23.7 \%$ of the markers from Plex-192 failed to genotype, this method proved to be a more time- and cost-effective means of isolating SNPs in a non-model species.

\subsection{Genotyping Success of GoldenGate Assays}

An overall (global) genotyping success rate of 85.4\% (41 SNPs) and 76.3\% (142 SNPs) was obtained for Plex-48 and Plex-192, respectively. Of the successfully genotyped loci, a total of 159 SNPs were found to be polymorphic and 24 were monomorphic (Table 2). These results were based on allele calls obtained with the GenomeStudio ${ }^{\text {TM }}$ Genotyping Module in which genotypes are generated for individuals at each locus as genoplots. A GenCall score was subsequently generated to represent the clustering of each individual SNP and, thus, the reliability of each genotyping score [45]. Scores ranged between zero and one and a score closer to one indicated that the genotype inferred was reliable, which could be visually verified with the genoplots (Figure 1). Lower GenCall scores were less reliable and indicated a clear separation from the center of the cluster. Failed SNPs could not be assigned to a genotypic cluster, due to low GenCall and GenTrain $(<0.25$ for Plex- $48 ;<0.45$ for Plex-192) scores depicted in Figure 1a. Sanger sequencing of randomly selected SNPs from Plex-48 confirmed the accuracy and reliability of the calls made by the GenomeStudio module. Polymorphic (Figure 1b) and monomorphic loci (Figure 1c) could also be distinguished through variation in the clustering. Only SNPs consisting of $\mathrm{a} \geq 0.80$ GenTrain score and $\mathrm{a} \geq 80 \%$ call rate, as well as a minor allele frequency (MAF) of $\geq 0.01$ were considered to be successfully genotyped.

Figure 1. Genoplots obtained with the GenomeStudio ${ }^{\mathrm{TM}}$ Genotyping Module representative of (a) a failed SNP; (b) a successfully genotyped (polymorphic) SNP; and (c) a monomorphic SNP analyzed in this study.

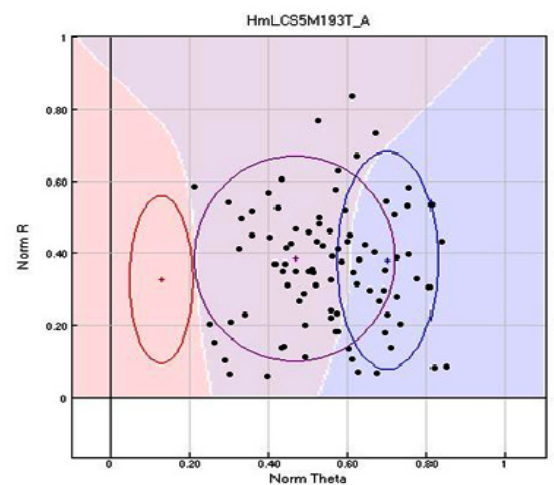

(a)

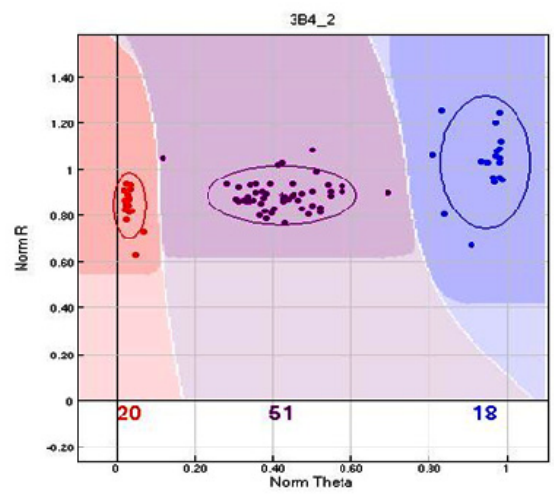

(b)

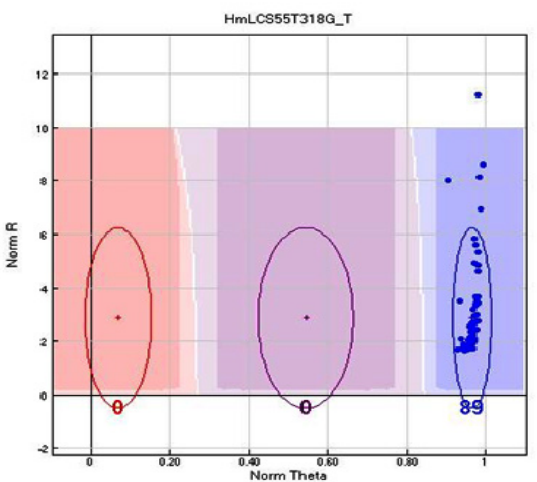

(c) 
In this study, only SNPs with a functionality score $\geq 0.75$ were selected for validation to ensure a high genotyping success rate, but despite this, 14.6\% (7 from Plex-48) and 22.9\% (44 from Plex-192) still failed to cluster and were considered genotyping failures. Previous studies found that the inclusion of SNPs with functionality scores $\leq 0.6$ reduced the overall success rate of such markers significantly $[13,46]$. In Plex-48, the recommended GenTrain cut-off $<0.25$ [47], indicating a low call rate, was utilized. For Plex-192, a more stringent GenTrain cut-off of $<0.45$ was used, while a GenCall rate $\geq 80 \%$ was applied in both assays.

The success of any genotyping method is reflected in what is referred to as the conversion rate and the global success rate. The former considers only the polymorphic markers, whereas the global success rate considers all the markers (monomorphic and polymorphic) that were successfully typed within the sample group [13]. In this study, a global success rate of $85.4 \%$ for Plex-48 and $76.3 \%$ for Plex-192 proved to be relatively high and corresponds to that obtained for other non-model species, such as, for example, catfish (Ictalurus spp. - 69\%; [44]) and maritime pine (Pinus pinaster - 66.9\%; [13]. The conversion rate obtained for Plex-48 (65.4\%) and Plex-192 (69.4\%) was also in accordance, e.g., with catfish (Ictalurus spp.-59.8\%; [41]) and the white- (Picea glauca-69.2\%) and black spruce (Picea mariana-77.1\%) [46]. The conversion rates for the current study fall short when compared to the manufacturers' predictions (93\%); however, it must be noted that these predictions are based on research in model species [48]. Factors that may have attributed to the lower conversion rates are the extreme GenTrain cut-off values applied in the current study, the presence of paralogous SNPs or limited knowledge regarding the complexity of the abalone genome.

Final confirmation of the success of the current genotyping assays is the significantly lower number of monomorphic SNPs (24\% and 7.5\%, for Plex-48 and Plex-192, respectively) that was found in comparison to other aquaculture species [41]. Interestingly, some markers were found to be heterozygous in all individuals, which could be due to cross-amplification of the allele-specific primers [36] and could represent paralogous duplications, a phenomenon already discovered in the sperm lysin gene in H. tuberculata coccinea [49]. These markers were not utilized in further applications.

Table 2. Comparison of the genotyping success of two GoldenGate assays (Plex-48 and Plex-192) assembled for H. midae. EST, expressed sequence tag.

\begin{tabular}{ccc}
\hline & Plex-48 & Plex-192 \\
\hline Functionality score & 0.75 & 0.8 \\
ESTs/Contigs & 35 & 139 \\
Feasible SNPs & 48 & 186 \\
GenTrain score & 0.25 & 0.45 \\
Total & 48 & 186 \\
Failures & $7(14.58 \%)$ & $44(23.7 \%)$ \\
Monomorphic & $10(20.83 \%)$ & $14(7.5 \%)$ \\
Polymorphic & $31(64.58 \%)$ & $128(68.8 \%)$ \\
\hline
\end{tabular}

\subsection{Functional Annotation and SNP Effect}

Functional annotation of the contigs utilized in the compilation of the GoldenGate assays (Plex-48 and Plex-192) indicated that the majority of the sequences showed significant similarity to the genes of 
interest: 88\% (Plex-48, Table 3) and 79\% (Plex-192). Further detail of annotation, sequence similarity (E-value), expected variants and SNP position and the effect for SNPs of Plex-48 and Plex-192 are shown in Supplementary Tables S1 and S2, respectively.

The majority of hits were classified in the Mollusca (47\%) and Chordata (22\%) phyla. The group Mollusca was further divided into Gastropoda and Bivalvia, with twice as many significant hits classed as Gastropoda. A total of 31 contigs could not be annotated, and the position and functional effect for four Plex-48 SNPs (8.33\%) and 30 Plex-192 SNPs (16.13\%) could, therefore, not be determined. For the two assays combined, $34.61 \%$ of the SNPs were located in the 3 ' untranslated regions (UTRs), while $50.85 \%$ of the SNPs were in coding regions. Further analysis of functional changes revealed that 69 of the total SNPs were synonymous and 50 were non-synonymous, of which the latter accounted for over $20 \%$ of the SNPs developed in this study and could be considered as functionally important changes in the corresponding proteins [50].

Table 3. Annotation, variants and predicted gene location and functional effect of the 48 feasible SNPs validated in the GoldenGate genotyping assay, Plex-48. UTR, untranslated region.

\begin{tabular}{|c|c|c|c|c|}
\hline SNP Name & EST/Contig & Functional annotation & Variant & SNP effect \\
\hline $3 B 4 \_2$ & \multirow{2}{*}{ 3B4 } & \multirow{2}{*}{ 60s Ribosomal protein L8 } & $\mathrm{T} / \mathrm{C}$ & UTR \\
\hline $3 B 4 \_7$ & & & $\mathrm{~A} / \mathrm{T}$ & UTR \\
\hline $3 D 10 \_1$ & $3 \mathrm{D} 10$ & Hemocyanin & $\mathrm{A} / \mathrm{G}$ & UTR \\
\hline $2 H 9 \_2$ & $2 \mathrm{H} 9$ & Ribosomal protein L22 & $\mathrm{A} / \mathrm{T}$ & UTR \\
\hline$H d S N P c 148 \_820 T \_C$ & HdSNPc148 & Actin & $\mathrm{T} / \mathrm{C}$ & Synonymous \\
\hline HdSNPc106_688C_T & HdSNPc106 & Tubulin alpha-1a chain isoform 2 & $\mathrm{C} / \mathrm{T}$ & Synonymous \\
\hline$H m S N P c 4 \_815 C \_T$ & HmSNPc4 & Microsatellite sequence & $\mathrm{C} / \mathrm{T}$ & Non-synonymous \\
\hline HaSNPdw500_207C_T & HaSNPdw & Microsatellite sequence & $\mathrm{C} / \mathrm{T}$ & UTR \\
\hline HmLCS5M193T_A & HmLCS5M & - & $\mathrm{T} / \mathrm{A}$ & - \\
\hline HmLCS5M479C_T & & Opacity protein & $\mathrm{C} / \mathrm{T}$ & Synonymous \\
\hline HmLCS55T318G_T & HmLCS55T & Microsatellite sequence & $\mathrm{G} / \mathrm{T}$ & Non-synonymous \\
\hline HmRS36T262T_C & HmRS36T & 5-Formyltetrahydrofolate cyclo-ligase & $\mathrm{T} / \mathrm{C}$ & Synonymous \\
\hline SNP101_113 & \multirow{2}{*}{ Contig 101} & \multirow{2}{*}{ Myosin heavy chain } & $\mathrm{A} / \mathrm{C}$ & UTR \\
\hline SNP101_201 & & & $\mathrm{C} / \mathrm{G}$ & UTR \\
\hline SNP146.2_132 & \multirow{2}{*}{ Contig 146} & \multirow{2}{*}{$\mathrm{ADP} / \mathrm{ATP}$ carrier protein } & $\mathrm{A} / \mathrm{G}$ & Synonymous \\
\hline SNP146.3_123 & & & $\mathrm{T} / \mathrm{G}$ & Non-synonymous \\
\hline SNP149.1_106 & \multirow{5}{*}{ Contig 149} & \multirow{5}{*}{ Heat shock protein 70} & $\mathrm{~A} / \mathrm{C}$ & UTR \\
\hline SNP149.1_374 & & & $\mathrm{C} / \mathrm{G}$ & Non-synonymous \\
\hline SNP149.2_165 & & & $\mathrm{A} / \mathrm{G}$ & Synonymous \\
\hline SNP149.4_75 & & & $\mathrm{A} / \mathrm{G}$ & UTR \\
\hline SNP149.4_341 & & & $\mathrm{T} / \mathrm{C}$ & UTR \\
\hline SNP210_266 & Contig 210 & - & $\mathrm{T} / \mathrm{G}$ & - \\
\hline SNP214_86 & \multirow{2}{*}{ Contig 214} & \multirow{2}{*}{ Ribosomal protein L10 } & $\mathrm{T} / \mathrm{C}$ & Non-synonymous \\
\hline SNP214_434 & & & $\mathrm{T} / \mathrm{C}$ & UTR \\
\hline SNP342.2_537 & Contig 342 & Heat shock protein & $\mathrm{T} / \mathrm{C}$ & UTR \\
\hline SNP449.2_110 & \multirow{2}{*}{ Contig 449} & \multirow{2}{*}{$\begin{array}{l}\text { s-Adenosylmethionine synthetase } \\
\text { isoform type-1 }\end{array}$} & $\mathrm{A} / \mathrm{G}$ & Non-synonymous \\
\hline SNP449.2_443 & & & $\mathrm{T} / \mathrm{C}$ & Synonymous \\
\hline
\end{tabular}


Table 3. Cont.

\begin{tabular}{|c|c|c|c|c|}
\hline SNP Name & EST/Contig & Functional annotation & Variant & SNP effect \\
\hline SNP1718_109 & Contig 1718 & NADH dehydrogenase subunit 1 & $\mathrm{~A} / \mathrm{T}$ & UTR \\
\hline SNP1833_160 & Contig 1833 & Alpha tubulin & $\mathrm{A} / \mathrm{G}$ & UTR \\
\hline SNP1834_76 & \multirow{2}{*}{ Contig 1834} & \multirow{2}{*}{ Tubulin alpha-1a chain- partial } & $\mathrm{A} / \mathrm{G}$ & UTR \\
\hline SNP1834_464 & & & $\mathrm{A} / \mathrm{G}$ & Non-synonymous \\
\hline SNP1949_235 & Contig 1949 & Ribosomal protein L3 & $\mathrm{A} / \mathrm{C}$ & UTR \\
\hline SNP4691_183 & Contig 4691 & Heat shock protein 70 & $\mathrm{~A} / \mathrm{G}$ & UTR \\
\hline SNP17550.1_463 & \multirow{3}{*}{ Contig 17550} & \multirow{3}{*}{ Clathrin heavy chain 1} & $\mathrm{~A} / \mathrm{G}$ & Non-synonymous \\
\hline SNP17550.3_221 & & & $\mathrm{A} / \mathrm{T}$ & UTR \\
\hline SNP17550.3_555 & & & $\mathrm{A} / \mathrm{T}$ & UTR \\
\hline SNP48_322 & Contig 48 & Collagen alpha-4 chain & $\mathrm{T} / \mathrm{G}$ & UTR \\
\hline SNP67_164 & Contig 67 & Collagen alpha-6 partial & $\mathrm{A} / \mathrm{G}$ & UTR \\
\hline SNP140_2421 & Contig 140 & $\mathrm{Na}^{+} \mathrm{K}^{+}$-ATPase alpha subunit & $\mathrm{T} / \mathrm{C}$ & Synonymous \\
\hline SNP229_2772 & Contig 229 & 14-3-3 Zeta & $\mathrm{T} / \mathrm{C}$ & UTR \\
\hline SNP300_1828 & Contig 300 & - & $\mathrm{A} / \mathrm{G}$ & - \\
\hline SNP972_1055 & Contig 972 & Myosin heavy chain & $\mathrm{T} / \mathrm{C}$ & Synonymous \\
\hline SNP1001_388 & Contig 1001 & Cre-sca-1 protein & $\mathrm{T} / \mathrm{C}$ & UTR \\
\hline SNP2091_264 & Contig 2091 & - & $\mathrm{A} / \mathrm{C}$ & - \\
\hline SNP3129_923 & Contig 3129 & Arginine kinase & $\mathrm{A} / \mathrm{G}$ & Synonymous \\
\hline SNP5837_204 & Contig 5837 & Mucus-associated protein partial & $\mathrm{T} / \mathrm{C}$ & Non-synonymous \\
\hline SNP13865_165 & Contig 13865 & Cathepsin 1 & $\mathrm{~T} / \mathrm{C}$ & UTR \\
\hline SNP20648_3041 & Contig 20648 & Filamin-c isoform 4 & $\mathrm{~A} / \mathrm{G}$ & Synonymous \\
\hline
\end{tabular}

\subsection{SNP Diversity, Population Differentiation and Family Informativeness}

All SNP loci showed two alleles and were in agreement with those originally observed before validation (Table 4, Table S3). Of the 159 polymorphic SNPs, another 13 were in fact monomorphic in the subsamples selected for estimation of genetic diversity parameters. Among the rest, MAF ranged from 0.0014 to 0.4781 for Plex-48 with a mean of 0.1542 (Table 4), while for Plex-192, MAF ranged from 0.0417 to 0.5 with a mean of 0.2086 (Table S3). Observed $\left(\mathrm{H}_{\mathrm{o}}\right)$ and unbiased expected heterozygosity $\left(\mathrm{H}_{\mathrm{e}}\right)$ values ranged from 0.003 to 0.788 and 0.003 to 0.497 , respectively, for Plex-48 and from 0.083 to 0.750 and 0.083 to 0.522 for Plex-192. For Plex-48, 15 SNP loci deviated significantly from Hardy-Weinberg equilibrium (HWE) $(p<0.05)$, while only 12 SNPs were not in accordance with HWE for Plex-192. The low to moderate levels of heterozygosity along with the average MAF (18.1\%) observed in the majority of the SNPs applied in this study were comparable to reports in, for example, Pacific abalone, H. discus hannai [17,18] and turbot, Scophthalmus maximus [51]. Although the average MAF was indicative of a fairly significant degree of homozygosity, a low inbreeding coefficient (average $f=-0.188$ ) was observed for all the Plex-48 SNPs and most of the SNPs of Plex-192. 
Table 4. Genetic diversity estimates of the polymorphic SNP markers (Plex-48) for H. midae individuals. HW, Hardy-Weinberg.

\begin{tabular}{|c|c|c|c|c|c|}
\hline \multirow{2}{*}{ SNP Name } & \multirow{2}{*}{$\begin{array}{c}\text { Minor allele } \\
\text { frequency }\end{array}$} & \multicolumn{2}{|c|}{ Heterozygosity } & \multirow{2}{*}{$\begin{array}{l}\text { Inbreeding } \\
\text { coefficient }\end{array}$} & \multirow{2}{*}{$\begin{array}{c}\text { Probability } \\
\text { HW } \\
\end{array}$} \\
\hline & & Observed & Expected & & \\
\hline $3 B 4 \_2$ & 0.4549 & 0.592 & 0.465 & -0.274 & 0.01 \\
\hline $3 B 4 \_7$ & 0.1901 & 0.537 & 0.473 & -0.134 & $<0.01$ \\
\hline 3D10_1 & 0.0391 & 0.281 & 0.472 & 0.406 & 0.001 \\
\hline HdSNPc148_820T_C & 0.4564 & 0.183 & 0.172 & -0.066 & 0.000 \\
\hline HdSNPc106_688C_T & 0.0015 & 0.721 & 0.462 & -0.563 & $<0.01$ \\
\hline HmRS36T262T_C & 0.0420 & 0.009 & 0.331 & 0.972 & 0.002 \\
\hline SNP101_113 & 0.0557 & 0.069 & 0.073 & 0.049 & 0.530 \\
\hline SNP101_201 & 0.0015 & 0.091 & 0.126 & 0.278 & 0.786 \\
\hline SNP146.2_132 & 0.1352 & 0.285 & 0.245 & -0.165 & 0.011 \\
\hline SNP149.1_374 & 0.0651 & 0.025 & 0.031 & 0.189 & 0.799 \\
\hline SNP149.2_165 & 0.4079 & 0.483 & 0.478 & -0.009 & 0.001 \\
\hline SNP149.4_75 & 0.2464 & 0.003 & 0.003 & 0.000 & - \\
\hline SNP210_266 & 0.0669 & 0.044 & 0.043 & -0.021 & 1.000 \\
\hline SNP214_86 & 0.0000 & 0.041 & 0.040 & -0.019 & 0.875 \\
\hline SNP342.2_537 & 0.1023 & 0.098 & 0.111 & 0.116 & 0.793 \\
\hline SNP449.2_110 & 0.4035 & 0.32 & 0.497 & 0.357 & 0.000 \\
\hline SNP1834_76 & 0.4781 & 0.013 & 0.012 & -0.005 & - \\
\hline SNP1834_464 & 0.0014 & 0.788 & 0.489 & -0.614 & $<0.01$ \\
\hline SNP1949_235 & 0.1764 & 0.309 & 0.266 & -0.162 & 0.000 \\
\hline SNP4691_183 & 0.0667 & 0.138 & 0.353 & 0.610 & 0.025 \\
\hline SNP17550.3_221 & 0.0189 & 0.013 & 0.013 & -0.005 & - \\
\hline SNP17550.3_555 & 0.0841 & 0.119 & 0.112 & -0.062 & 0.081 \\
\hline SNP67_164 & 0.0696 & 0.182 & 0.176 & -0.033 & 0.181 \\
\hline SNP140_2421 & 0.1272 & 0.178 & 0.162 & -0.096 & 0.310 \\
\hline SNP229_2772 & 0.0338 & 0.099 & 0.128 & 0.228 & 0.880 \\
\hline SNP300_1828 & 0.0678 & 0.195 & 0.181 & -0.075 & 0.058 \\
\hline SNP972_1055 & 0.2267 & 0.082 & 0.394 & 0.794 & $<0.01$ \\
\hline SNP1001_388 & 0.1893 & 0.280 & 0.250 & -0.119 & 0.000 \\
\hline SNP2091_264 & 0.2580 & 0.314 & 0.486 & 0.354 & 0.001 \\
\hline SNP3129_923 & 0.2246 & 0.318 & 0.462 & 0.312 & $<0.01$ \\
\hline SNP20648_3041 & 0.0889 & 0.164 & 0.166 & 0.015 & 0.063 \\
\hline
\end{tabular}

-no $p$-value was assigned.

Applying the 31 polymorphic markers of Plex-48, population differentiation between three wild and three commercial populations was inferred based on summary statistics (Wrights' $F$-statistics), as well as multivariate analysis (FCA). Pairwise $F_{\mathrm{ST}}(\theta)$ values ranged from 0.006 to 0.199 , with significance $(p<0.05)$ over all but one of the population pairs tested. Results indicated limited genetic differentiation between the three wild populations, while the relatively high values $\left(F_{\mathrm{ST}}=0.090-0.140\right)$ obtained between wild and cultured populations indicated considerable genetic differentiation between wild and cultured abalone. Factorial correspondence analysis was performed to obtain a three-dimensional view of the genetic relationship between the six different populations. The first 
factor accounted for $43.39 \%$ of the genetic variation and the second factor for $24.02 \%$. Most evident from the FCA plots was the tight clustering of the three wild populations and the noticeable genetic distinctness of each of the three cultured populations (Figure 2). Comparison of the individual genotypes supported the substantial overlap of individuals from wild populations, while virtually no overlapping was observed between individuals of the three cultured populations. Population analysis indicated that allele fixation (increasing $F_{\mathrm{ST}}$ ) was evident between cultured and wild populations, but was most striking between the three cultured full-sib families. The differentiation between wild and cultured abalone was in accordance with what has recently been found in H. midae based on microsatellite markers [3], while the unusually high variation observed between the cultured populations (pairwise $F_{\mathrm{ST}}>0.15$ ) could be attributed to the highly heterozygous nature of the wild-caught parents, leading to an increase in the probability of the resulting F1 progenies receiving two different alleles at each locus, inflating the genetic distinctness of the respective families. With regards to the wild populations that included abalone from the West (Saldanha Bay), South (Witsand) and East coast (Riet Point) of South Africa, FCA results corroborated the summary statistics in that the only statistically unsupported pairwise $F_{\mathrm{ST}}$ value was found between the wild populations of Witsand and Saldanha Bay. Bester-van der Merwe et al. [52] previously reported that there was low, yet significant, differentiation between wild populations of $H$. midae, but that the primary barrier to gene flow was around the Cape-Agulhas area. Saldanha Bay is situated west of Cape-Agulhas and Witsand and Saldanha Bay, east of the proposed barrier. The level of population differentiation and pattern of gene flow depicted by the SNPs in this study are therefore in full agreement with the population structure previously suggested for wild $H$. midae populations. The population differentiation was also supported by the molecular analysis of variance (AMOVA) results, with significant differentiation amongst populations within groups $\left(F_{\mathrm{SC}}=0.141, p=0.000\right)$ and within populations $\left(F_{\mathrm{ST}}=0.141\right.$, $p=0.000)$.

Mendelian segregation analysis showed that only a small percentage of markers did not adhere to Mendelian patterns of inheritance in the respective mapping families, and the highest percentage (20\%) was exhibited for family DS_1. Only a very small number of SNPs (19 across all families) showed distortion of segregation after correction for multiple tests, and most of the markers were distorted in one family only. Consequently, a high percentage of the SNPs (118) developed in this study were found to be informative markers (heterozygous in both or at least one of the parents) and could be considered as candidates for linkage map construction [53]. However, due to their bi-allelic nature, a larger number of SNP markers versus microsatellites should be included in a linkage mapping study, and these markers should initially be genotyped in the parents to ensure that less of these markers will need to be excluded. This would ensure the inclusion of only informative markers in linkage map construction without the need to discard monomorphic markers. In addition, testing for non-Mendelian segregation of the SNPs is also useful in detecting the presence of null alleles [50]. According to the Mendelian ratios obtained in this study, possible null alleles were present for only a small percentage of markers, confirming their usefulness in future applications. 
Figure 2. Factorial component analysis (FCA) based on 31 SNP loci in six H. midae populations. The respective populations are indicated by color: Family DS_1 (Yellow), Family DS_5 (Blue), Family DS_6 (White), Riet Point (Grey), Saldanha Bay (Pink) and Witsand (Green). The wild populations are encircled.

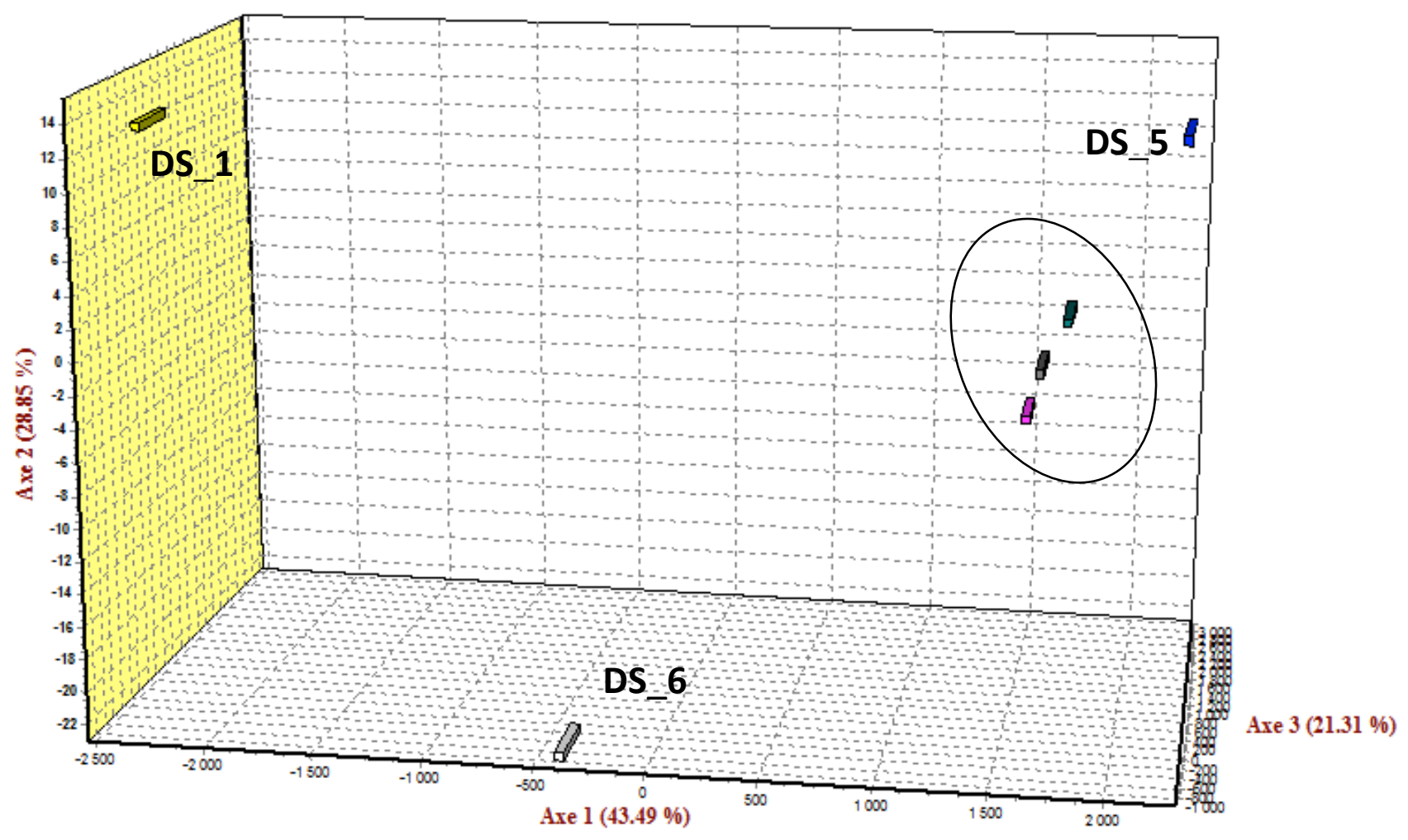

\section{Experimental Section}

\subsection{Transcriptome Sequencing and SNP discovery}

Samples and methods for sequencing and de novo assembly of the $H$. midae transcriptome have been described in detail by Franchini et al. [44]. In brief, this included RNA extraction from 19 animals from a single family, followed by cDNA library construction and sequence generation by the Illumina Genome Analyzer (GA II). For EST characterization and identification of SNPs, contig assembly and annotation were performed utilizing two separate bioinformatic analyses. The first analysis involved the use of Velvet 0.7 .52 for contig assembly and the cDNA Annotation system (dCAS) 1.4.3 for annotation of contigs. In the second analysis, high quality reads were assembled de novo using the CLC Genomics Workbench v4.0 software (CLCbio, Aarhus, Denmark), and sequence annotation was performed using Blast2GO 2.4.4 [54]. In both analyses, the databases against which the annotation was completed included the eukaryote clusters of genes (KOG; [55]), Gene Ontology (GO; [56]) and the Kyoto Encyclopedia of Genes and Genomes (KEGG; [57-59]).

For the in vitro identification of SNPs, annotated contigs resulting from the first bioinformatic analysis were screened manually to identify contigs with significant hits $\left(E\right.$-value $\left.<1.0 \times 10^{-17}\right)$ against genes with known functions using the BLASTN algorithm (BLAST) [60]. A set of 58 annotated contigs $\left(E\right.$-value $\left.<1.3 \times 10^{-19}\right)$ were selected and facilitated the design of 97 primer pairs with BatchPrimer3 [61]. Twenty of the 58 contigs were fragmented into smaller sections of 
approximately $700 \mathrm{bp}$ to ensure amplification of the entire contig with internal primers. Successful primers were used to amplify the genomic DNA of eight unrelated $H$. midae individuals in $10 \mu \mathrm{L}$ reaction volumes containing $20 \mathrm{ng}$ genomic DNA, $200 \mu \mathrm{M}$ dNTPs, $2.0 \mathrm{mM} \mathrm{MgCl} 2,2.0$ pmol of each primer and $0.25 \mathrm{U} \mathrm{GoTaq}^{\circledR}$ Flexi DNA polymerase (Promega, Madison, WI, USA). Thermal cycling was conducted on the Gene-Amp System 2700 thermal cycler (Applied Biosystems, Foster City, CA, USA), and conditions consisted of an initial denaturing step of $95^{\circ} \mathrm{C}$ for $5 \mathrm{~min}$, followed by 35 cycles of $94{ }^{\circ} \mathrm{C}$ for $30 \mathrm{~s}$, Tm (specific for each primer pair) for $45 \mathrm{~s}, 72{ }^{\circ} \mathrm{C}$ for $45 \mathrm{~s}$ and a final elongation step of $10 \mathrm{~min}$ at $72{ }^{\circ} \mathrm{C}$. Assessment of PCR amplification was conducted through $2 \%$ agarose gel electrophoresis. PCR amplicons were purified, quantified and Sanger sequenced using the BigDye ${ }^{\circledR}$ Terminator v3 Cycle Sequencing kit (Applied Biosystems) and the ABI PRISM ${ }^{\circledR} 3100$ DNA automated sequencer (Applied Biosystems). DNA sequence chromatograms were individually analyzed using Sequence Scanner v1.0 (Applied Biosystems) to determine sequence quality. Multiple alignments for sequences of each primer pair were carried out using ClustalW v1.4 [62], implemented in BioEdit v7.0.0 [63]. Sequence similarity was determined with BLASTN to assess if the correct amplicons were amplified. Alignments and chromatograms of all eight individuals were visually screened for putative SNPs. Only base positions with two peaks, of which the height ratio was approximately $\geq 1: 2$, were considered as putative SNP loci for heterozygous individuals.

For the in silico identification of SNPs, the SNP detection module in CLC Genomics Workbench was used to screen 22,761 assembled contigs following the second bioinformatic pipeline. The parameters that were set for putative SNP calling included a minimum quality score of 20 , minimum coverage of 80 and a minor allele frequency (MAF) $>10 \%$. Of these, 139 SNP-containing contigs were selected for further analysis of 400 putative SNPs. To ensure reliable primer design, the flanking sequences of putative SNPs were checked to ensure that there were no other polymorphisms within $60 \mathrm{bp}$ of the SNPs. Contigs containing the predicted SNPs were then subjected to BLAST similarity searches using Blast2GO.

\subsection{SNPs and Samples for GoldenGate Assays}

SNP characterization using the GoldenGate genotyping assay was conducted in two separate multiplex assays, further referred to as Plex-48 and Plex-192. The SNPs selected for Plex-48 were comprised of 24 in vitro SNPs developed in the first bioinformatic analysis, 12 in vitro SNPs previously developed by Bester et al. (2008) and Rhode (2010) and 12 novel in silico SNPs identified by means of the second bioinformatic pipeline using CLC Genomics Workbench. The in silico SNPs represented a test panel to determine the efficiency and performance of markers identified with the CLC workbench. For Plex-192, 186 SNPs from the second bioinformatic pipeline and six SNP loci from Plex-48 (positive SNP controls) were included. For both assays, sequences containing putative SNPs were submitted to the Illumina Assay Design Tool to determine the functionality and primer designability score for each SNP locus. Scores of $0.5-1.0$ are required for a high quality assay, and only loci with a final score $\geq 0.75$ were considered for genotyping.

Samples for Plex-48 included individuals from three wild abalone populations from the coast of South Africa (Saldanha Bay, Witsand and Riet Point), as well as parents and offspring of four linkage mapping families collected from two commercial abalone farms (Table 5). The samples used for 
Plex-192 included individuals from six linkage mapping families reared on two commercial farms, two of which overlapped with the cultured populations used in Plex-48 (Table 5). DNA samples were precipitated and resuspended in TE Buffer ( $1 \mathrm{M}$ Tris-HCl, $0.5 \mathrm{M}$ EDTA, water at $\mathrm{pH}$ 8.0) or DNase-free water (Promega, Madison, WI, USA) to a final concentration of $50 \mathrm{ng} / \mu \mathrm{L}$. Each plate from Plex-48 contained two positive and one negative control to monitor genotyping efficiency and possible contamination, and plates from Plex-192 each contained 3 genotyping (positive) controls.

Table 5. Sample sizes of $H$. midae populations genotyped in Plex-48 and Plex-192.

\begin{tabular}{ccc}
\hline \multirow{2}{*}{ Sample origin } & \multicolumn{2}{c}{ Number of individuals } \\
& Plex-48 & Plex-192 \\
\hline Family DS 1 & 103 & 70 \\
Family DS_2 & 94 & 87 \\
Family DS_5 & 90 & - \\
Family DS_6 & 94 & - \\
Family D & - & 72 \\
Family H & - & 71 \\
Family I & - & 81 \\
Family J & - & 72 \\
Saldanha Bay & 23 & - \\
Witsand & 26 & - \\
Riet Point & 26 & - \\
Positive controls & 2 per plate & 3 per plate \\
Negative controls & - & 1 per plate \\
\hline
\end{tabular}

\subsection{SNP Genotyping and Functional Effect}

Genotyping was achieved with the Illumina GoldenGate genotyping assay according to the manufacturer's specifications. Genotyping was conducted at the National Health Laboratory Services (NHLS) facility at the University of the Witwatersrand, South Africa. The GenomeStudio ${ }^{\mathrm{TM}}$ Genotyping Module v1.0 was employed for data analysis to infer genotypes and allele frequencies for each locus. Data quality assessment was done with the default No-Call (GenCall) parameters defined by Illumina, a GenTrain (clustering algorithm) value of 0.25 for Plex-48 and 0.45 for Plex-192 and an individual call rate of 0.85 for Plex-48 and 0.80 for Plex-192. The more stringent GenTrain value used for Plex-192 was due to a lack of clustering taking place at values below 0.45, which led to the exclusion of those markers. Individuals that failed genotyping and SNPs that illustrated ambiguous clustering were omitted prior to further analysis. As an additional means of validation, nine randomly selected SNPs were sequenced in 30 individuals using the ABI PRISM ${ }^{\circledR}$ BigDye Terminator v3.1 Cycle Sequencing kit in the forward direction. Genotypes acquired from the Sanger sequencing were manually compared to those generated by the Genotyping Module. For one of the cultured populations, DS_2, the majority (87.6\%) of the population could not be genotyped, due to technical difficulties, and were excluded from further analysis.

All the ESTs and contigs screened for SNPs in this study were subjected to BLASTX similarity searches against the NCBI protein database using Blast2GO. Functionally annotated contigs with the highest sequence similarity were used to identify the strand and reading frame orientation. The contig 
sequences containing SNPs within coding regions, or, alternatively, the corresponding $120 \mathrm{bp}$ flanking sequences, were translated with the EMBOSS transeq utility for all six possible reading frames. The resulting amino acid sequences for alternative alleles at each SNP locus were compared to determine whether SNPs were synonymous or non-synonymous (Tables S1 and S2).

\subsection{Genetic Diversity, Population and Family Analysis}

All successfully genotyped SNPs were further evaluated for the level of polymorphism observed in a subset of individuals that represented wild abalone samples. For Plex-48, this included all the wild populations and the parents of the four full-sib families, while for Plex-192, only the 12 parents (six males and six females) of the six mapping families were included, as they were the only individuals of Plex-192 that could be considered wild abalone. Genetic diversity estimates, such as minor allele frequency, observed and expected heterozygosity and locus-specific $F_{\text {is, }}$, were obtained using GENEPOP 4.0 [64]. Hardy-Weinberg equilibrium (HWE) was also computed via the exact probability test (10,000 dememorizations, 500 batches and 5000 iterations per batch) in GENEPOP.

In order to test for population differentiation between wild and cultured populations (including offspring), the polymorphic markers from Plex-48 were employed to determine the pairwise $F_{\text {ST }}$ estimator $(\theta)$ of Weir and Cockerham [65] in GENETIX 4.04 [66]. Significance was tested with 1000 permutations, and the Bonferroni correction model was used to adjust the significance levels for multiple tests. Population allele frequency data were subjected to factorial component analysis (FCA), also available in GENETIX. This provided a three-dimensional view of the distribution of genetic variation between the six genotyped populations. Molecular analysis of variance (AMOVA, 10,000 permutations) was performed in ARLEQUIN 3.5.1 to further test the grouping hypothesis of wild versus cultured populations.

Selected progenies of all six mapping families (two of which were analyzed with both Plex-48 and Plex-192) included in this study were used to evaluate conformance to Mendelian segregation for all the polymorphic SNPs (234). Offspring ranged from 70 individuals for Family $\mathrm{H}$ to 103 for Family DS_1. SNP markers were tested for segregation distortion from expected Mendelian ratios with the chi square goodness-of-fit test $(p<0.05)$ and subjected to linkage analysis using JOINMAP ${ }^{\circledR} 4.1$ [67] to create sex-average and sex-specific maps. Linkage analysis and results are reported in detail by Vervalle et al. [53].

\section{Conclusions}

The major objective of this study was to investigate the utility of ESTs generated by Illumina sequencing-by-synthesis for the development of SNPs in the abalone, Haliotis midae, since transcriptome sequencing in non-model species promises greater representation of the functional characteristics of these species [30,68,69]. The increased popularity of type I markers has redirected research toward the development of SNPs instead of microsatellites. These SNPs can ultimately be applied in conjunction with microsatellite markers in various applications, such as genetic diversity studies, population structure analyses, linkage mapping, quantitative trait locus (QTL) analysis and parentage assignment. A further objective of this study was therefore to test the utility of the successfully genotyped SNPs in determining genetic diversity, population differentiation and family 
informativeness in $H$. midae, since previously, these types of applications were addressed mainly using microsatellite markers $[3,5,55,70]$. In this study, transcriptome characterization with the aid of NGS technologies proved to be adequate for the use of marker development in $H$. midae. The Illumina GoldenGate assay was equally successful in testing the utility of these markers in population differentiation inference, as well as in the saturation of a preliminary linkage map for H. midae, both of which are very important in the genetic management of this South African mollusk. In the current study, contigs for SNP development were selected based on a vast selection of genes of relevance, ranging from cellular processes to stress response, which is potentially also linked to important traits in aquaculture species, such as disease resistance and growth. Further analysis of these markers, specifically focusing on the functional SNPs identified in this study, can be directed to target genes of interest, such as those regulating immune response and environmental adaptation, as well as gene expression studies to facilitate a better understanding of this species' genome.

\section{Acknowledgments}

We thank Britt Drögemöller for reading through the draft of the manuscript and performing English corrections. We are also grateful to Clint Rhode for communicating this work at an international symposium on aquaculture genetics. This study was supported by a research grant from the National Research Foundation, the Innovation Fund and Industry partners (Irvin \& Johnson Limited, Abagold (Pty) Ltd, Aquafarm Development Company (Pty) Ltd., HIK Abalone (Pty) Ltd. and Roman Bay Sea Farm (Pty) Ltd.). Stellenbosch University is thanked for the facilities provided and B. Godfrey, G. Harkins and G. Isaacs for assistance in sample collection.

\section{Conflicts of Interest}

The authors declare no conflict of interest.

\section{References}

1. An, H.S.; Lee, J.W.; Kim, H.C.; Myeong, J.-I. Genetic characterization of five hatchery populations of the pacific abalone (Haliotis discus hannai) using microsatellite markers. Int. J. Mol. Sci. 2011, $12,4836-4849$.

2. Department of Agriculture Forestry and Fisheries. Aquaculture Annual Report 2011, March 2012, p. 15. Available online: http://www.nda.agric.za/doaDev/fisheries/ (accessed on 17 June 2013).

3. Rhode, C.; Hepple, J.; Jansen, S.; Davis T.; Vervalle, J.; Bester-van der Merwe A.E.; Roodt-Wilding, R. A Population genetic analysis of abalone domestication events in South Africa: Implications for the management of the abalone resource. Aquaculture 2012, 356, 235-242.

4. Bester, A.E.; Slabbert, R.; D’Amato, M.E. Isolation and characterisation of microsatellite markers in the South African abalone (Haliotis midae). Mol. Ecol. Resour. 2004, 4, 618-619.

5. Slabbert, R.; Ruivo, N.R.; Van den Berg, N.C.; Lizamore, D.L.; Roodt-Wilding, R. Isolation and characterisation of 63 microsatellite loci for the abalone Haliotis midae. J. World Aquacult. Soc. 2008, 39, 429-435. 
6. Slabbert, R.; Hepple, J.; Venter, A.; Nel, S.; Swart, L.; Van den Berg, N.C.; Roodt-Wilding, R. Isolation and inheritance of 44 microsatellite loci in the South African abalone Haliotis midae L. Anim. Genetics 2010, 41, 332-333.

7. Slabbert, R.; Hepple, J.-A.; Rhode, C.; Bester-Van der Merwe, A.E.; Roodt-Wilding, R. New microsatellite markers for the abalone Haliotis midae developed by 454 pyrosequencing and in silico analyses. Gen. Mol. Res. 2012, 11, 2769-2779.

8. Bester, A.E.; Roodt-Wilding, R.; Whitaker, H.A. Discovery and evaluation of single nucleotide polymorphisms (SNPs) for Haliotis midae: A targeted EST approach. Anim. Genetics 2008, 39, 321-324.

9. Rhode, C.; Slabbert, R.; Roodt-Wilding, R. Microsatellite flanking regions: A SNP mine in South African abalone (Haliotis midae). Anim. Genetics 2008, 39, 329.

10. Lepoittevin, C.; Frigerio, J.M.; Garnier-Gere, P.; Salin, F.; Cervera, M.T.; Vornam, B.; Harvengt, L.; Plomion, C. In vitro vs. in silico detected SNPs for the development of a genotyping array: What can we learn from a non-model species? PLoS One 2010, 5, e11034.

11. Hubert, S.; Bussey, J.T.; Higgins, B.; Curtis, B.A.; Bowman, S. Development of single nucleotide polymorphism markers for Atlantic cod (Gadus morhua) using expressed sequences. Aquaculture 2009, 296, 7-14.

12. Moen, T.; Hayes, B.; Nilsen, F.; Delghandi, M.; Fjalestad, K.T.; Fevolden, S.E.; Berg, P.R.; Lien, S. Identification and characterisation of novel SNP markers in Atlantic cod: Evidence for directional selection. BMC Genetics 2008, 9, 18.

13. Liu, W.; Li, H.; Bao, X.; He, C.; Li, W.; Shan, Z. The first set of EST-derived single nucleotide polymorphism markers for Japanese scallop Patinopecten yessoensis. J. World Aquacult. Soc. 2011, 42, 456-461.

14. Andreassen, R.; Lunner, S.; Hoyheim, B. Targeted SNP discovery in Atlantic salmon (Salmo salar) genes using a 3'UTR-primed SNP detection approach. BMC Genomics 2010, 11, 706.

15. Hayes, B.; Laerdahl, J.K.; Lien, S.; Moen, T.; Berg, P.; Hindar, K.; Davidson, W.S.; Koop, B.F.; Adzhubei, A.; Hoyheim, B. An extensive resource of single nucleotide polymorphism markers associated with Atlantic salmon (Salmo salar) expressed sequences. Aquaculture 2007, 265, 82-90.

16. Liu, S.; Zhou, Z.; Lu, J.; Sun, F.; Wang, S.; Liu, H.; Jiang, Y.; Kucuktas, H.; Kaltenboeck, L.; Peatman, E.; et al. Generation of genome-scale gene-associated SNPs in catfish for the construction of a high-density SNP array. BMC Genomics 2011, 12, 53.

17. Qi, H.; Liu, X.; Zhang, G.; Wu, F. Mining expressed sequences for single nucleotide polymorphisms in Pacific abalone (Haliotis discus hannai). Aquac. Res. 2009, 40, 1661-1667.

18. Qi, H.; Liu, X.; Wu, F.; Zhang, G. Development of gene-targeted SNP markers for genomic mapping in Pacific abalone Haliotis discus hannai Ino. Mol. Biol. Rep. 2010, 37, 3779-3784.

19. Buetow, K.H.; Edmonson, M.N.; Cassidy, A.B. Reliable identification of large numbers of candidate SNPs from public EST data. Nat. Genetics 1999, 21, 323-325.

20. Gurvey, V.; Berezikov, E.; Malik, R.; Plasterk, R.H.A.; Cuppen, E. Single nucleotide polymorphism associated with rat expressed sequences. Genome Res. 2004, 14, 1438-1443.

21. Hayes, B.J.; Nilsen, K.; Berg, P.R.; Grindflek, E.; Lien, S. SNP detection exploiting multiple sources of redundancy in large EST collections improves validation rates. Bioinformatics 2007, $23,1692-1693$. 
22. Bouck, A.; Vision, T. The molecular ecologist's guide to expressed sequence tags. Mol. Ecol. 2007, 16, 907-924.

23. Wang, S.; Peatman, E.; Abernathy, J.; Waldbieser, G.; Lindquist, E.; Richardson, P.; Lucas, S.; Wang, M.; Li, P.; Thimmapuram, J.; et al. Assembly of 500,000 inter-specific catfish expressed sequence tags and large scale gene-associated marker development for whole genome association studies. Catfish Genome Consortium. Genome Biol. 2010, 11, R8.

24. Metzker, M.L. Applications of next-generation sequencing: Sequencing technologies-The next generation. Nat. Rev. Genetics 2010, 11, 31-46.

25. Van Bers, N.E.M.; Van Oers, K.; Kerstens, H.H.D.; Dibbits, B.W.; Crooijmans, R.P.; Visser, M.E.; Groenen, M.A. SNP detection in the great tit Parus major using high throughput sequencing. Mol. Ecol. 2010, 19, 89-99.

26. Kerstens, H.H.D.; Crooijmans, R.P.M.A.; Veenendaal, A.; Dibbits, B.W.; Chin-A-Woenq, T.F.; Den Dunnen, J.T.; Groenen, M.A. Large scale single nucleotide polymorphism discovery in unsequenced genomes using second generation high throughput sequencing technology: Applied to turkey. BMC Genomics 2009, 10, 479.

27. Renaut, S.; Nolte, A.W.; Bernatchez, L. Mining transcriptome sequences towards identifying adaptive single nucleotide polymorphisms in lake whitefish species pairs (Coregonus spp. Salmonidae). Mol. Ecol. 2010, 19, 115-131.

28. Stapley, J.; Reger, J.; Feulner, P.G.D.; Smadja, C.; Galindo, J.; Ekblom, R.; Bennison, C.; Ball, A.D.; Beckerman, A.P.; Slate, J. Adaptation genomics: The next generation. Trends Ecol. Evol. 2010, 25, 705-712.

29. Le Dantec, L.; Chagné, D.; Pot, D.; Cantin, O.; Garnier-Géré, P.; Bedon, F.; Frigerio, J.M.; Chaumeil, P.; Léger, P.; Garcia, V.; et al. Automated SNP detection in expressed sequence tags: Statistical considerations and application to maritime pine sequences. Plant Mol. Biol. 2004, 54, 461-470.

30. Diopere, E.; Hellemans, B.; Volckaert, F.A.M.; Maes, G.E. Identification and validation of single nucleotide polymorphisms in growth- and maturation-related candidate genes in sole (Solea solea L.). Mar. Genomics 2013, 9, 33-38.

31. Useche, F.J.; Gao, G.; HanaFey, M.; Rafalski, A. High-Throughput Identification Database Storage and Analysis of SNPs in EST Sequences. Genome Inform. 2001, 12, 194-203.

32. Garvin, M.R.; Saitoh, K.; Gharrett, A.J. Application of single nucleotide polymorphisms to non-model species: A technical review. Mol. Ecol. Resour. 2010, 10, 915-934.

33. Fan, J.B.; Gunderson, K.L; Bibikova, M.; Yeakley, J.M.; Chen, J.; Wickhamgarcia, E.; Lebruska, L.; Laurent, M.; Shen, R.; Barker, D. Illumina universal bead arrays. Methods Enzymol. 2006, 410, 57-73.

34. Illumina. GoldenGate ${ }^{\circledR}$ Genotyping with VeraCode ${ }^{\mathrm{TM}}$ Technology: Custom 96-plex and 384-plex Assays. 2008. Available online: http://www.illumina.com/ (accessed on 28 June 2013).

35. Illumina. VeraCode Technology. 2010. Avalaible online: http://www.illumina.com/ (accessed on 28 June 2013).

36. Zhang, L.S.; Guo, X.M. Development and validation of single nucleotide polymorphism markers in the eastern oyster Crassostrea virginica Gmelin by mining ESTs and resequencing. Aquaculture 2010, 302, 124-129. 
37. Bai, Z.; Yin, Y.; Hu, S.; Wang, G.; Zhang, X.; Li, J. Identification of genes involved in immune response, microsatellite, and SNP markers from Expressed Sequence Tags generated from hemocytes of freshwater pearl mussel (Hyriopsis cumingii). Mar. Biotechnol. 2009, 11, $520-530$.

38. Kang, J-H.; Appleyard, S.A.; Elliot, N.G.; Jee, Y-J.; Lee, J.B.; Kang, S.W.; Baek, M.K.; Han, Y.S.; Choi, T.J.; Lee, Y.S. Development of genetic markers in abalone through construction of a SNP database. Anim. Genetics 2011, 42, 309-315.

39. Kim, W-J.; Jung, H.; Gaffney, P. Development of type I genetic markers from expressed sequence tags in highly polymorphic species. Mar. Biotechnol. 2010, 13, 1-6.

40. Scofield, D.G.; Hong, X.; Lynch, M. Position of the final intron in full-length transcripts: Determined by NMD? Mol. Biol. Evol. 2007, 24, 896-899.

41. Wang, S.L.; Sha, Z.X.; Sonstegard, T.S.; Liu, H.; Xu, P.; Somridhivej, B.; Peatman, E.; Kucuktas, H.; Liu, Z.J. Quality assessment parameters for EST-derived SNPs from catfish. BMC Genomics 2008, 9, 450.

42. Jonker, R.M.; Zhang, Q.; Van Hooft, P.; Loonen, M.J.; Van der Jeugd, H.P.; Crooijmans, R.P.; Groenen, M.A; Prins, H.H.; Kraus, R.H. The development of a genome wide SNP set for the Barnacle Goose Branta leucopsis. PLoS One 2012, 7, e38412.

43. Eckert, A.J.; Pande, B.; Ersoz, E.S.; Wright, M.H.; Rashbrook, V.K.; Nicolet, C.M.; Neale, D.B. High-throughput genotyping and mapping of single nucleotide polymorphisms in loblolly pine (Pinus taeda L.). Tree Genet. Genomes 2009, 5, 225-234.

44. Franchini, P.; Van der Merwe, M.; Roodt-Wilding, R. Transcriptome characterization of the South African abalone Haliotis midae using sequencing-by-synthesis. BMC Res. Notes 2011, 4, 59.

45. Shen, R.; Fan, J.B.; Campbell, D.; Chang, W.; Chen, J.; Doucet, D.; Yeakley, J.; Bibikova, M.; Wickham Garcia, E.; McBride, C.; et al. High-throughput SNP genotyping on universal bead arrays. Mutat. Res. 2005, 573, 70-82.

46. Pavy, N.; Pelgas, B.; Beauseigle, S.; Blais, S.; Gagnon F.; Gosselin I.; Lamothe M.; Isabel N.; Bousquet, J. Enhancing genetic mapping of complex genomes through the design of highly-multiplexed SNP arrays: Application to the large and unsequenced genomes of white spruce and black spruce. BMC Genomics 2008, 9, 21.

47. Fan, J.B.; Oliphant, A.; Shen, R.; Kermani, B.G.; Garcia, F.; Gunderson, K.L.; Hansen, M.; Steemers, F.; Butler, S.L.; Deloukas, P.; et al. Highly parallel SNP genotyping. Cold Spring Harb. Symp. Quant. Biol. 2003, 68, 69-78.

48. Montpetit, A.; Nelis, M.; Laflamme, P.; Magi, R.; Ke, X.; Remm, M.; Cardon, L.; Hudson, T.J.; Metspalu, A. An evaluation of the performance of tag SNPs derived from HapMap in a Caucasian population. PLoS Genet. 2006, 2, 282-290.

49. Clark, N.L.; Findlay, G.D.; Yi, X.; MaCoss, M.J.; Swanson, W.J. Duplication and selection on abalone sperm lysin in an allopatric population. Mol. Biol. Evol. 2007, 24, 2081-2090.

50. Vera, M.; Alvarez-Dios, J.A.; Millan, A.; Pardo, B.G.; Bouza, C.; Hermida, M.; Fernandez, C.; De la Herran, R.; Molina-Luzon, M.J.; Martinez, P. Validation of single nucleotide polymorphism (SNP) markers from an immune Expressed Sequence Tag (EST) turbot; Scophthalmus maximus; database. Aquaculture 2011, 313, 31-41. 
51. Vera, M.; Alvarez-Dios, J.-A.; Fernandez, C.; Bouza, C.; Vilas, R.; Martinez, P. Development and validation of single nucleotide polymorphisms (SNPs) markers from two transcriptome 454-runs of turbot (Scophthalmus maximus) using high-throughput genotyping. Int. J. Mol. Sci. 2013, 14, 5694-5711.

52. Bester-van der Merwe, A.E.; Roodt-Wilding, R.; Volckaert, F.A.M.; D’Amato, M.E. Historical isolation and hydrodynamically constrained gene flow in declining populations of the South African abalone Haliotis midae. Conserv. Genet. 2011, 12, 543-555.

53. Vervalle, J.; Hepple, J.; Jansen, S.; Du Plessis, J.; Wang, P.; Rhode, C.; Roodt-Wilding, R. Integrated linkage map of Haliotis midae Linnaeus based on microsatellites and SNPs. J. Shellfish Res. 2013, 32, 89-103.

54. Conesa, A.; Gotz, S.; Garcia-Gomez, J.M.; Terol, J.; Talon, M.; Robles, M. Blast2GO: A universal tool for annotation visualization and analysis in functional genomics research. Bioinformatics 2005, 21, 3674-3676.

55. Tatusov, R.L.; Fedorova, N.D.; Jackson, J.D.; Jacobs, A.R.; Kiryutin, B.; Koonin, E.V.; Krylov, D.M.; Mazumder, R.; Mekhedov, S.L.; Nikolskaya, A.N.; et al. The COG database: An updated version includes eukaryotes. BMC Bioinform. 2003, 4, 41.

56. Ashburner, M.; Ball, C.A.; Blake, J.A.; Botstein, D.; Butler, H.; Cherry, J.M.; Davis, A.P.; Dolinski, K.; Dwight, S.S.; Eppig, J.T.; et al. Gene ontology: Tool for the unification of biology. The Gene Ontology Consortium. Nat. Genet. 2000, 25, 25-29.

57. Kanehisa, M.; Goto, S. KEGG: Kyoto encyclopedia of genes and genomes. Nucl. Acids Res. 2000, 28, 27-30.

58. Kanehisa, M.; Goto, S.; Hattori, M.; Aoki-Kinoshita, K.F.; Itoh, M.; Kawashima, S.; Katayama, T.; Araki, M.; Hirakawa, M. From genomics to chemical genomics: New developments in KEGG. Nucl. Acids Res. 2006, 34, 354-357.

59. Kanehisa, M.; Goto, S.; Furumichi, M.; Tanabe, M.; Hirikawa, M. KEGG for representation and analysis of molecular networks involving diseases and drugs. Nucl. Acids Res. 2010, 38, 355-360.

60. Altschul, S.F.; Gish, W.; Miller, W.; Myers, E.W.; Lipman, D.J. Basic local alignment search tool. J. Mol. Biol. 1990, 215, 403-410.

61. You, F.M.; Huo, N.; Gu, Y.Q.; Luo, M.C.; Ma, Y.; Hane, D.; Lazo, G.R.; Dvorak, J.; Anderson, O.D. BatchPrimer3: A high throughput web application for PCR and sequencing primer. BMC Bioinform. 2008, 9, 253.

62. Thompson, J.D.; Higgins, D.G.; Gibson, T.J. CLUSTAL W improving the sensitivity of progressive multiple sequence alignment through sequence weighting; position-specific gap penalties and weight matrix choice. Nucl. Acids Res. 1994, 22, 4673-4680.

63. Hall, T.A. BioEdit: A user-friendly biological sequence alignment editor and analysis program fro Windows 95/98/NT. Nucl. Acids Symp. Ser. 1999, 41, 95-98.

64. Rousset, F. GENEPOP'007: A complete re-implementation of the GENEPOP software for Windows and Linux. Mol. Ecol. Resour. 2008, 8, 103-106.

65. Weir, B.S.; Cockerham, C.C. Estimation F-statistics for the analysis of population structure. Evolution 1984, 38, 1358-1370.

66. Genetix, Logiciel Sous WindowsTM Pour la Génétique des Populations, version 4.04; Laboratoire Génome et populations, CNRS UPR 9060, Université de Montpellier II: Montpellier, France, 2002. 
67. JoinMap ${ }^{\circledR}$, version 4; Software for the calculation of genetic linkage maps in experimental populations. Kyazma B.V. Wageningen, The Netherlands, 2006.

68. Surget-Groba, Y.; Montoya-Burgos, J.I. Optimization of de novo transcriptome assembly from next-generation sequencing data. Genome Res. 2010, 20, 1432-1440.

69. Quinn, N.L.; Levenkova, N.; Chow, W.; Bouffard, P.; Boroevich, K.A.; Knight, J.R.; Jarvie, T.P.; Lunieniecki, K.P.; Desany, B.A.; Koop, B.F.; et al. Assessing the feasibility of GS FLX Pyrosequencing for sequencing the Atlantic salmon genome. BMC Genomics 2008, 9, 404.

70. Slabbert, R.; Bester, A.E.; D'Amato, M.E. Analyses of genetic diversity and parentage within a South African hatchery of the abalone Haliotis midae Linnaeus using microsatellite markers. J. Shellfish Res. 2009, 28, 369-375.

(C) 2013 by the authors; licensee MDPI, Basel, Switzerland. This article is an open access article distributed under the terms and conditions of the Creative Commons Attribution license (http://creativecommons.org/licenses/by/3.0/). 